\title{
Acknowledgement to Reviewers of Axioms in 2015
}

\author{
Axioms Editorial Office \\ Published: 25 January 2016 \\ MDPI AG, Klybeckstrasse 64, CH-4057 Basel, Switzerland; axioms@mdpi.com
}

The editors of Axioms would like to express their sincere gratitude to the following reviewers for assessing manuscripts in 2015.

We greatly appreciate the contribution of expert reviewers, which is crucial to the journal's editorial decision-making process. Several steps have been taken in 2015 to thank and acknowledge reviewers. Good, timely reviews are rewarded with a discount off their next MDPI publication. By creating an account on the submission system, reviewers can access details of their past reviews, see the comments of other reviewers, and download a letter of acknowledgement for their records. In addition, MDPI has launched a collaboration with Publons, a website that seeks to publicly acknowledge reviewers on a per journal basis. This is all done, of course, within the constraints of reviewer confidentiality. Feedback from reviewers shows that most see their task as a voluntary and mostly unseen work in service to the scientific community. We are grateful to our reviewers for the contribution they make.

Alai, Daniel H.

Albouy, Pierre-Antoine

Angstmann, C. N.

Außenhofer, Lydia

Bagis, N.D.

Ben-El-Mechaiekh, Hichem

Beneduci, Roberto

Bergou, Janos

Breaz, Daniel Valer

Burlaga, L. F.

Chaichian, Masud

Chantawansri, Tanya L.

Chasco, M. J.

Chipchakov, Ivan

Choi, Junesang

Clayton, J. D.

Conte, Elio

Croix, Michael La

Croke, Sarah

Dehmer, Matthias

Dikranjan, D.

Ding, Jiu

Domínguez, Xabier

Dürr, Detlef

Feret, Jérôme

Gabriyelyan, Saak

Gazeau, Jean Pierre

Ghirardi, Gian Carlo
Hazewinkel, Michiel

He, Ying

Heinosaari, Teiko

Hofmann, Karl H.

Huang, Wenwen

Jahnert, F.

Jorgensen, Palle E. T.

Kearnes, Keith

Knill, Oliver

Kozubowski, Tomasz J.

Leiderman, Arkady G.

Luchko, Yury

Machado, Jose

Mainardi, Francesco

Maroney, Owen

Martini, Alessio

Matsumoto, Diogo Kendy

Matthes, Roland

McGreevy, John

McLennan, Andrew

Megrelishvili, Michael

Metzler, Ralf

Morita, Takeshi

Moschopoulos, Panagis

Münzenmaier, Steffen

Murty, M. Ram

Neeb, Karl-Hermann

Néel, M. C.
Oussa, Vignon S.

Pagnini, Gianni

Paris, Richard B.

Patané, Giuseppe

Provost, Serge B.

Renner, Renato

Rodrigues, M. M.

Rogosin, Sergei

Rump, Wolfgang

Sayas, Francisco-Javier

Scanlon, Thomas

Schlaak, Tobias

Schlosser, Michael

Shlossberg, Menachem

Tammer, C.

Tarieladze, Vaja

Temme, Nico M.

Tkachenko, Michael

Tumulka, Roderich

Twining, Carole

Tyson, Jon

Vaz, Sandra

Vourdas, Apostol

Walkden, Charles

Whitfield, James Daniel

Wüthrich, Christian

$\mathrm{Xu}$, Daoyi

Yamagata, Koichi 
Goldman, William

Gomez, Sergio

Gorenflo, Rudolf
Nickolas, Peter

Nieto, Juan J.

Orsingher, Enzo
Yoshida, Kentaroh

Zhang, Yang

Ziman, Mario

(C) 2016 by the author; licensee MDPI, Basel, Switzerland. This article is an open access article distributed under the terms and conditions of the Creative Commons by Attribution (CC-BY) license (http://creativecommons.org/licenses/by/4.0/). 